\title{
Volumetric analysis of chin and mandibular retromolar region as donor sites for cortico-cancellous bone blocks
}

\author{
Zeltner, Marco ; Flückiger, Laura B ; Hämmerle, Christoph H F ; Hüsler, Jürg ; Benic, Goran I
}

\begin{abstract}
AIM To test whether the mandibular retromolar region renders different results from the chin region with respect to the amount of bone available for the harvesting of block grafts. MATERIAL AND METHODS Sixty cone beam computed tomography (CBCT) scans of mandibles of adult patients without pathologic findings in the chin and retromolar region were included. According to the number of mandibular teeth, 20 CBCT data sets were allocated to each of the following groups: group M1: dentition 36-46; group M2: dentition 37-47; and group M3: dentition 38-48. For the potential donor sites in the chin and the retromolar regions, the volume (VChin, VRetro ), the length (LChin, LRetro ), the height (HChin, HRetro ) and the width (HChin , HRetro ) were assessed using a computer software. Moreover, the chin was examined for the presence and the localization of the mandibular incisive canal. To compare the donor sites in the chin and in the retromolar regions, the quotients VRetro /VChin, LRetro /LChin, HRetro /HChin and WRetro /WChin were calculated and tested using the Wilcoxon signed-rank test or the sign test. RESULTS The mean bone volume VChin measured $3.5 \pm 1.3 \mathrm{~cm}(3)$ (SD), whereas the overall VRetro amounted to $1.8 \pm 1.1 \mathrm{~cm}(3)$ (SD). VRetro amounted to $2.6 \pm 1.4 \mathrm{~cm}(3)$ (SD) in the group M1, $1.8 \pm 0.5 \mathrm{~cm}(3)$ in the group M2 and $1.0 \pm 0.4 \mathrm{~cm}(3)$ in the group M3. For the group M1, VRetro /VChin measured $82 \pm 39 \%(\mathrm{P}=0.036)$. VRetro /VChin reached $57 \pm 20 \%$ in the group $\mathrm{M} 2$ and $32 \pm 12 \%$ in the group M3 $(\mathrm{P}<0.001)$. The mandibular incisive canal was detected in $97 \%$ of the CBCT scans. The distance between the mandibular incisive canal and the apices of the central incisors measured $10.5 \pm 3.5 \mathrm{~mm}$. CONCLUSION The amount of bone available for the harvesting of cortico-cancellous blocks in the chin region was superior in comparison with the mandibular retromolar region. In the absence of the second and the third molars, the amount of bone harvestable in the retromolar region reached approximately $80 \%$ of the bone volume available in the chin region. In the majority of the cases, the mandibular incisive canal was detected within the donor site in the chin region.
\end{abstract}

DOI: https://doi.org/10.1111/clr.12746

Posted at the Zurich Open Repository and Archive, University of Zurich

ZORA URL: https://doi.org/10.5167/uzh-127134

Journal Article

Accepted Version

Originally published at:

Zeltner, Marco; Flückiger, Laura B; Hämmerle, Christoph H F; Hüsler, Jürg; Benic, Goran I (2016). Volumetric analysis of chin and mandibular retromolar region as donor sites for cortico-cancellous bone blocks. Clinical Oral Implants Research, 27(8):999-1004.

DOI: https://doi.org/10.1111/clr.12746 
1 Volumetric analysis of chin and mandibular

2 retromolar region as donor sites for cortico-

3 cancellous bone blocks

4

5 Marco Zeltner ${ }^{1}$, Laura B. Flückiger ${ }^{1}$, Christoph H.F. Hämmerle ${ }^{1}$, Jürg Hüsler ${ }^{2}$, Goran

$6 \quad$ I. Benic $^{1}$

7

$8{ }^{1}$ Clinic of Fixed and Removable Prosthodontics and Dental Material Science, Center of

9 Dental Medicine, University of Zurich, Zurich, Switzerland

$10{ }^{2}$ Institute of Mathematical Statistics and Actuarial Science, University of Bern, Bern,

11 Switzerland

12

13 Key words: bone, bone augmentation, graft, block, cone beam computed tomography,

14 CBCT, chin, symphysis, retromolar, ramus, donor site, radiology, volume

15

16 Running title: Chin vs. retromolar donor sites for bone blocks

17

18

19 Corresponding author:

20 Dr. Goran I. Benic

21 Clinic of Fixed \& Removable Prosthodontics and Dental Material Science

22 University of Zürich

23 Plattenstrasse 11

248032 Zürich

25 Switzerland

26 Tel: +41446343452

27 Fax: +41446344305

28 e-mail: goran.benic@zzm.uzh.ch 


\section{Abstract}

2 Aim: To test whether the mandibular retromolar region renders different results from the chin

3 region with respect to the amount of bone available for the harvesting of block grafts.

\section{Materials and Methods:}

5 Sixty cone beam computed tomography (CBCT) scans of mandibles without pathologic

6 findings in the chin and retromolar region of adult patients were included. According to the

7 number of mandibular teeth, $20 \mathrm{CBCT}$ data sets were allocated to each of the following

8 groups: group M1: dentition 36-46, group M2: dentition 37-47, group M3: dentition 38-48.

9 For the potential donor sites in the chin and the retromolar regions, the volume $\left(\mathrm{V}_{\text {Chin }}, \mathrm{V}_{\text {Retro }}\right)$,

10 the length $\left(\mathrm{L}_{\text {Chin }}, \mathrm{L}_{\text {Retro }}\right)$, the height $\left(\mathrm{H}_{\mathrm{Chin}}, \mathrm{H}_{\text {Retro }}\right)$ and the width $\left(\mathrm{H}_{\text {Chin }}, \mathrm{H}_{\text {Retro }}\right)$ were assessed

11 by using a computer software. Moreover, the chin was examined for the presence and the

12 localisation of the mandibular incisive canal. To compare the donor sites in the chin and in the

13 retromolar regions, the quotients $\mathrm{V}_{\text {Retro }} / \mathrm{V}_{\text {Chin, }} \mathrm{L}_{\text {Retro }} / \mathrm{L}_{\text {Chin }}, \mathrm{H}_{\text {Retro }} / \mathrm{H}_{\text {Chin }}$ and $\mathrm{W}_{\text {Retro }} / \mathrm{W}_{\text {Chin }}$ were

14 calculated and tested by using the Wilcoxon signed-rank test or the Sign test.

15 Results: The mean bone volume $\mathrm{V}_{\text {chin }}$ measured $3.5 \pm 1.3 \mathrm{~cm}^{3}(\mathrm{SD})$, whereas the overall $\mathrm{V}_{\text {Retro }}$ 16 amounted to $1.8 \pm 1.1 \mathrm{~cm}^{3}$ (SD). $\mathrm{V}_{\text {Retro }}$ amounted to $2.6 \pm 1.4 \mathrm{~cm}^{3}$ (SD) in the group M1, $1.8 \pm 0.5$ $17 \mathrm{~cm}^{3}$ in the group M2 and $1.0 \pm 0.4 \mathrm{~cm}^{3}$ in the group M3. For the group M1 $\mathrm{V}_{\text {Retro }} / \mathrm{V}_{\text {Chin }}$ 18 measured $82 \pm 39 \%(\mathrm{p}=0.055) . \mathrm{V}_{\text {Retro }} / \mathrm{V}_{\text {Chin }}$ reached $57 \pm 20 \%$ in the group $\mathrm{M} 2$ and $32 \pm 12 \%$ in 19 the group M3 $(\mathrm{p}<0.001)$. The mandibular incisive canal was detected in $97 \%$ of the CBCT 20 scans. The distance between the mandibular incisive canal and the apices of the central 21 incisors measured $10.5 \pm 3.5 \mathrm{~mm}$.

22 Conclusions: The amount of bone available for harvesting of cortico-cancellous blocks in the 23 chin region was superior in comparison to the mandibular retromolar region. In the absence of 24 the second and the third molars the amount of bone harvestable in the retromolar region 25 reached approximately $80 \%$ of the bone volume available in the chin region. In the majority 
1 of the cases the mandibular incisive canal was detected within the donor site in the chin

2 region.

3

4 


\section{Introduction}

Dental implants are used to replace missing teeth by anchoring dental prosthesis in situations of partial or complete edentulism. In jaw regions with reduced ridge dimensions precluding the primary stability of the implant in the prosthetically correct position, the staged approach for bone regeneration and implant placement may be required. Moreover, primary bone augmentation may be indicated in situations in which an unfavorable appearance of the soft tissue is expected owing to the lack of hard tissue support.

Autogenous bone blocks, alone, or in combination with bone substitute and/or collagen membranes, are the most reliable and successful procedures for staged horizontal and vertical augmentations of extended bone defects (Benic \& Hammerle 2014, Jensen \& Terheyden 2009).

Several intra- and extraoral donor sites for harvesting autogenous bone blocks were described in the literature, e.g. chin, mandibular retromolar region, iliac crest, calvarium (Lundgren, et al. 1996, Misch 1997).

The selection of the region for bone harvesting depends on the size and the localization of the bone defect at the recipient site. Intraoral donor sites can provide a sufficient amount of bone for the augmentation of localized alveolar ridge defects (Cordaro, et al. 2002, Jensen \& Sindet-Pedersen 1991, Misch \& Misch 1995). The benefit of this approach is the fact that the treatment can be performed under local anaesthesia, remaining limited to an intraoral surgical field (Raghoebar, et al. 2001).

Postoperative complications related to the intraoral bone harvesting include pain, swelling, bleeding, wound dehiscences, loss of tooth vitality, and temporary or permanent neurosensory disturbances (Hoppenreijs, et al. 1992, Nkenke, et al. 2001, von Arx, et al. 2005). A recent systematic review analysed comparative studies on the harvesting of autogenous bone grafts (Nkenke \& Neukam 2014). It was found that the mandibular ramus 
1 was the source of bone that was preferred by the patients. The patients' acceptance of chin

2 bone harvesting was low, owing to the fact that it caused a considerable morbidity at the

3 donor site. The investigators concluded that the retromolar mandibular area is, whenever

4 possible, the preferable site for intraoral harvesting of autogenous bone blocks. The findings

5 regarding the amount of bone available for the intraoral harvesting were limited.

6 The primary objective of the present cone beam computed tomography (CBCT) study

7 was to test whether or not the retromolar mandibular region renders similar results as the chin

8 region with respect to the amount of bone available for the harvesting of cortico-cancellous

9 bone blocks. Moreover, the study aimed to assess the presence and the localization of the

10 mandibular incisive canal in the mandibular symphysis region.

11 


\section{Materials and Methods}

\section{CBCT selection}

Sixty CBCT scans of mandibles in adult patients were derived from the database of the

Center of Dental Medicine, University of Zurich, Zurich, Switzerland. The CBCT database

was screened by one investigator according to the date of CBCT acquisition, starting with the most recent data set. Depending on the number of mandibular teeth, $20 \mathrm{CBCT}$ data sets were allocated to each of the following three groups:

- Group M1: dentition 31-36 and 41-46 / missing teeth: 37, 38, 47, 48

- Group M2: dentition 31-37 and 41-47 / missing teeth: 38 and 48

- Group M3: dentition 31-38 and 41-48 / complete mandibular dentition

The presence of pathologic findings in the chin and in the mandibular retromolar region was considered as an exclusion criterion. After the inclusion, the $\mathrm{CBCT}$ data sets were anonymised.

CBCT scans had been acquired with a 3DExam CBCT device (KaVo Dental, Biberich, Deutschland) and generated using the following technical parameters: $120 \mathrm{kV}$ acceleration voltage, $5 \mathrm{~mA}$ beam current, field of view (FOV) diameter of $16 \mathrm{~cm}, 360^{\circ}$ rotation, voxel size of 0.3 or $0.4 \mathrm{~mm}$ and scan time of $9 \mathrm{sec}$. The FOV included either mandible and maxilla, or the mandible only.

\section{CBCT analysis}

CBCT DICOM data sets were imported in the Simplant Pro implant planning software version 13.0 (Materialise Dental, Hasselt, Belgium). Prior to the analysis, a panoramic curve was drawn in the axial reconstruction. In each $\mathrm{CBCT}$ image, the boundaries of the potential donor sites in the chin and in both retromolar regions were determined in the panoramic, in the axial and in the bucco-oral reconstructions every 0.8 or $0.9 \mathrm{~mm}$. 
The region-of-interest (ROI) in the chin was determined by drawing the following

2 margins (Figures 1 and 2):

3

- Cranial: $5 \mathrm{~mm}$ apical to the root apices of the mandibular teeth

- Caudal: $2 \mathrm{~mm}$ cranial to the inferior border of the mandible

- Distal: $5 \mathrm{~mm}$ mesial to the mental foramens

- Buccal: buccal surface of the mandible

- Lingual: $2 \mathrm{~mm}$ to the lingual surface of the mandible.

The ROI in the retromolar areas were determined by drawing the following boundaries (Figure 3):

- Cranial: cranial surface of the mandible

- Caudal: $2 \mathrm{~mm}$ to the inferior alveolar canal

- Mesial: $2 \mathrm{~mm}$ distal to the most distal molar

- Distal: area perpendicular to the inferior alveolar canal crossing through the intersection between the cranial surface of the mandible and the first bucco-oral CBCT reconstruction with a consistently visible ascending ramus

- Buccal: buccal surface of the mandible

- Lingual: $2 \mathrm{~mm}$ buccal to the lingual surface of the mandible.

The following parameters were assessed:

- Volume in the chin $\left(\mathrm{V}_{\text {chin }}\right)$ and in the retromolar regions $\left(\mathrm{V}_{\text {retro }}\right)$ (Figure 4$)$

- Length (mesio-distal dimension) in the chin $\left(\mathrm{L}_{\text {chin }}\right)$ and in the retromolar regions

$$
\text { ( } \left.\mathrm{L}_{\text {retro }}\right)
$$

- Width (bucco-oral dimension) in the chin $\left(\mathrm{W}_{\text {chin }}\right)$ (mean value of width measurements apical to the mandibular lateral incisors) and in the retromolar regions $\left(\mathrm{W}_{\text {retro }}\right)$ (mean value of width measurements at the mesial and the distal borders of ROI) 
- Height (cranio-caudal dimension) in the chin $\left(\mathrm{H}_{\text {chin }}\right)$ (in the mandibular symphysis) and in the retromolar regions $\left(\mathrm{H}_{\text {retro }}\right)$ (mean value of height measurements at the mesial and the distal borders of the ROI).

Additionally, the chin region was examined for the presence and the localisation of the mandibular incisive canal. The distance between the apices of the mandibular central incisors and the mandibular incisive canal was assessed in the bucco-oral image reconstructions in a direction perpendicular to the axial plane. The results of the two measurements were averaged to one value per patient. One investigator selected the CBCT data sets and performed the measurements.

\section{Statistical analysis}

The results from the left and right retromolar regions were averaged to one value per patient. Descriptive statistics were computed for all the parameters (SAS 9.4, SAS Institute Inc., Cary, NC, USA). The data were reported by using means, standard deviations (SD), ranges, medians, lower, and upper quartiles. To compare the potential donor sites at the chin and the retromolar regions, the quotients $\mathrm{V}_{\text {Retro }} / \mathrm{V}_{\text {Chin }}, \mathrm{L}_{\text {Retro }} / \mathrm{L}_{\text {Chin }}, \mathrm{H}_{\text {Retro }} / \mathrm{H}_{\text {Chin }}$ and $\mathrm{W}_{\text {Retro }} / \mathrm{W}_{\text {Chin }}$ were calculated and tested with reference to the value 1 . The Wilcoxon signed-rank test and the confidence intervals (CI) for a Hodges-Lehman estimate for the median were applied to detect a difference between the median and the value 1 . If the assumption of symmetry could not be confirmed, the Sign test and its corresponding confidence interval (CI) were used. Results of tests with p-values $\leq 0.05$ were considered statistically significant. 


\section{Results}

Group M1 comprised CBCT scans of 11 female and 9 male patients, whereas groups

M2 and M3 included CBCT scans of 10 female and 10 male patients each. The mean patient age amounted to $56.8 \pm 13.1$ years (SD) in the group M1, $46.3 \pm 17.1$ years (SD) in the group M2 and 30.6 \pm 9.7 years (SD) in the group M3.

The results of volume and linear measurements for the chin and the mandibular retromolar regions are summarized in Table 1.

The mean harvestable bone volume $\mathrm{V}_{\text {chin }}$ measured $3.4 \pm 1.5 \mathrm{~cm}^{3}$ (SD) in the group M1 (median: $3.4 \mathrm{~cm}^{3}$ ), $3.4 \pm 0.9 \mathrm{~cm}^{3}(\mathrm{SD})$ in the group M2 (median: $3.4 \mathrm{~cm}^{3}$ ), and $3.6 \pm 1.4$ $\mathrm{cm}^{3}$ (SD) in the group M3 (median: $3.3 \mathrm{~cm}^{3}$ ). $V_{\text {Retro }}$ amounted to $2.6 \pm 1.4 \mathrm{~cm}^{3}$ (SD) in the group M1 (median: $2.4 \mathrm{~cm}^{3}$ ), $1.8 \pm 0.5 \mathrm{~cm}^{3}(\mathrm{SD})$ in the group M2 (median: $1.8 \mathrm{~cm}^{3}$ ) and $1.1 \pm$ $0.4 \mathrm{~cm}^{3}$ (SD) in the group M3 (median: $1.0 \mathrm{~cm}^{3}$ ). The corresponding quotient $\mathrm{V}_{\text {Retro }} / \mathrm{V}_{\text {Chin }}$ reached $82 \pm 39 \%$ (SD) for the group M1 (median: $79 \%$, CI: $63-99 \%$ ) , $57 \pm 20 \%$ (SD) for the group M2 (median: $58 \%$, CI: $46-66 \%$ ) and $32 \pm 12 \%$ (SD) for the group M3 (median: $30 \%$, CI: $26-38 \%$ ). In all the groups the quotients $\mathrm{V}_{\text {Retro }} / \mathrm{V}_{\text {Chin }}$ were statistically significant $(\mathrm{p}<0.05)$ indicating that the amount of bone in the mandibular retromolar region was significantly lower in comparison to the chin (Table1).

When considering the linear measurements, $\mathrm{L}_{\text {Retro }} / \mathrm{L}_{\text {Chin }}$ reached $99 \pm 25 \%$ (SD) for the group M1 (median: $93 \%$, CI: $81-116 \%$ ), $67 \pm 15 \%$ (SD) for the group M2 (median: 67 $\%$, CI: $59-75 \%$ ) and $39 \pm 10 \%$ (SD) for the group M3 (median: 41\%, CI: $35-44 \%$ ). The quotient $\mathrm{W}_{\text {Retro }} / \mathrm{W}_{\text {Chin }}$ amounted to $90 \pm 17 \%$ (SD) for the group M1 (median: $90 \%$, CI: 81 $98 \%$ ), $82 \pm 19 \%$ (SD) for the group M2 (median: $80 \%$, CI: $73-91 \%$ ) and $84 \pm 22 \%$ (SD) for the group M3 (median: $82 \%$, CI: 73 - $93 \%$ ). With regards to the cranio-caudal dimension, $\mathrm{H}_{\text {Retro }} / \mathrm{H}_{\text {Chin }}$ measured $91 \pm 57 \%$ (SD) for the group M1 (median: $70 \%$, CI: $65-$ 
$1108 \%$ ), $87 \pm 26 \%$ (SD) for the group M2 (median: $77 \%$, CI: $77-98 \%$ ) and $87 \pm 21 \%$

2 (SD) for the group M3 (median: $90 \%, \mathrm{CI}: 77-98 \%$ )

The mandibular incisive canal was detected in $97 \%(58 / 60)$ of the evaluated CBCT

4 scans. The mean distance between the mandibular incisive canal and the apices of the

5 mandibular central incisors in the apico-coronal direction measured $10.5 \pm 3.5 \mathrm{~mm}$ (SD)

6 (minimum: $3.5 \mathrm{~mm}$, maximum: $20.3 \mathrm{~mm}, 95 \% \mathrm{CI}: 9.58-11.42 \mathrm{~mm}$ ).

7 


\section{Discussion}

The present study showed that the amount of bone for harvesting of cortico-cancellous

3 blocks in the chin region was superior in comparison to the mandibular retromolar region. In

4 the absence of the second and the third molars, the amount of bone harvestable in the

5 retromolar region reached approximately $80 \%$ of the volume available in the chin region.

A previous study assessed the amount of harvestable bone in the mandibular symphysis by analysing 15 CT scans (Yavuz, et al. 2009). The anatomical boundaries applied for the definition of the donor site were the same as the ones used in the present investigation. The calculated bone volume was $3.5 \pm 0.7 \mathrm{~cm}^{3}$ and the average size of the block measured 39 $\mathrm{mm} \times 11 \mathrm{~mm} \times 8 \mathrm{~mm}$. In a recent examination the volume of bone graft that can be harvested from the mandibular symphysis and rami was analysed on $40 \mathrm{CT}$ scans (Verdugo, et al. 2014). The CAD calculation yielded a harvestable bone volume of $1.4 \pm 0.5 \mathrm{~cm}^{3}$ for the symphysis and $0.8 \pm 0.2 \mathrm{~cm}^{3}$ for each ramus. The amount of bone harvested in the chin and the mandibular retromolar region was assessed in a clinical study with 50 patients (Misch 1997). The intra-surgical assessment revealed that the graft volume from the chin was approximately the double in comparison to the retromolar region $\left(1.7 \mathrm{~cm}^{3}\right.$ vs. $\left.0.9 \mathrm{~cm}^{3}\right)$, mainly result of increased thickness of the grafts. The differences in the absolute values between the studies can be explained by different anatomical boundaries of the donor sites and by different analytical methods.

There are several techniques for bone harvesting in the mandibular retromolar region. A procedure with an extension of the retromolar donor site to include the ascending ramus and the buccal cortex caudal to the alveolar canal was described (Clavero \& Lundgren 2003, Soehardi, et al. 2009). Two examinations on human cadavers measured the size of block grafts obtained from the ascending ramus and the chin (Brockhoff, et al. 2014, Yates, et al. 2013). The volume and the area of block grafts obtained from the ramus measured in average 
1 the double in comparison to the mandibular symphysis. In a recent clinical study, primary

2 ridge augmentations with bone blocks obtained from the ascending rami were performed in 314 patients with atrophic edentulous maxillae (Hernandez-Alfaro, et al. 2013). In 5 out of the

414 cases, the inferior alveolar nerve was exposed during graft elevation. After 14 to 16 weeks

5 of healing, the investigators found adequate bone volume for implant placement. It was,

6 therefore, concluded that the ascending ramus offers a sufficient amount of bone for extended 7 augmentation of edentulous maxillae.

In the present investigation, the mandibular retromolar donor region was determined by applying a safety distance of $2 \mathrm{~mm}$ to the alveolar canal. Moreover, the donor region was defined aiming at a bone block with a maximal thickness. In contrast, the previously mentioned procedure for bone harvesting from the ascending ramus consists of the removal of the buccal cortex (Soehardi, et al. 2009).

In the current study, the mandibular incisive canal was detected in $97 \%$ of the examined CBCT scans within the chin donor site. This finding is in agreement with the recent CT examinations, in which the mandibular incisive canal was found in the majority of the patients examined (Makris, et al. 2010, Romanos, et al. 2012). Several studies reported that autogenous bone harvesting from the chin region is related to higher postoperative morbidity and number of complications, in comparison with autogenous bone harvesting from the retromolar region (Cordaro, et al. 2011, Silva, et al. 2006). The high rate of postoperative sensory disturbances and loss of tooth vitality after bone harvesting from the mandibular symphysis can be explained by the damage of the blood vessels and nerve bundles in the anterior mandible (von Arx, et al. 2005, Weibull, et al. 2009). Modern surgical devices for piezoelectric osteotomy allow to selectively cut the bone and to reduce the risk of damage of the soft tissue structures (Pereira, et al. 2014, Sohn, et al. 2007). Therefore, the complication rates after bone harvesting with piezoelectric instruments may differ from the rates found in the previous studies using conventional instruments. 
The main limitation of the present study is the fact that the same investigator selected

2 the CBCT data sets and performed the non-blinded measurements. The risk of bias can, 3 therefore, not be excluded. However, efforts were made to standardize the CBCT

4 measurements by applying consistent margins for the definition of the regions of interest.

When selecting the site for intra-oral harvesting of autogenous bone, the amount of

6 bone needed for grafting and the risk of complications should be considered. The chin 7 generally offers a larger bone volume for harvesting in comparison to the mandibular 8 retromolar region. However, large interindividual variability exists regarding the amount of

9 bone that can be harvested, and this is determined by the location of anatomical boundaries 10 such as teeth, blood vessels and nerve bundles. Owing to the potentially lower risk of 11 complications, the mandibular retromolar area is, whenever possible, the preferable site for 12 intra-oral harvesting of autogenous bone blocks. 


\section{Conclusions}

2 Within the limitations of the present study, it can be concluded that for harvesting of cortico-

3 cancellous bone blocks:

4

5

6

7

8

9

10

11

12

\section{Acknowledgements}

14 This study was supported by the Clinic of Fixed and Removable Prosthodontics and Dental

15 Material Science, Center of Dental Medicine, University of Zurich, Switzerland.

16

17

18

19

20

- The amount of harvestable bone in the chin region was superior in comparison to the mandibular retromolar region.

- In the absence of the second and the third molars, the amount of bone in the retromolar region reached approximately $80 \%$ of the bone volume available in the chin region.

- In the majority of the cases the mandibular incisive canal was detected within the bone donor site in the chin region. 


\section{Figure legend}

2 Figure 1: Bucco-oral reconstruction of the region-of-interest in the chin area 3 5

8 Figure 4: Three-dimensional reconstruction of the regions-of-interest in the chin and the

9 retromolar area

10

11 


\section{Table legend}

4 Table 1: Results of volume, length, width and height of donor sites in the chin and the 5 retromolar region for different study groups

6

7 


\section{$1 \quad$ References}

Benic, G. I. \& Hammerle, C. H. (2014) Horizontal bone augmentation by means of guided bone regeneration. Periodontology 2000 66: 13-40.

Brockhoff, H. C., 2nd, Yates, D. M., Finn, R. \& Phillips, C. (2014) Comparison of intraoral harvest sites in the edentulous versus dentate specimen. Oral Surg Oral Med Oral Pathol Oral Radiol 117: 575-580.

Clavero, J. \& Lundgren, S. (2003) Ramus or chin grafts for maxillary sinus inlay and local onlay augmentation: Comparison of donor site morbidity and complications. Clinical Implant Dentistry and Related Research 5: 154-160.

Cordaro, L., Amade, D. S. \& Cordaro, M. (2002) Clinical results of alveolar ridge augmentation with mandibular block bone grafts in partially edentulous patients prior to implant placement. Clinical Oral Implants Research 13: 103-111.

Cordaro, L., Torsello, F., Miuccio, M. T., di Torresanto, V. M. \& Eliopoulos, D. (2011) Mandibular bone harvesting for alveolar reconstruction and implant placement: Subjective and objective cross-sectional evaluation of donor and recipient site up to 4 years. Clinical Oral Implants Research 22: 1320-1326.

Hernandez-Alfaro, F., Sancho-Puchades, M. \& Guijarro-Martinez, R. (2013) Total reconstruction of the atrophic maxilla with intraoral bone grafts and biomaterials: A prospective clinical study with cone beam computed tomography validation. International Journal of Oral and Maxillofacial Implants 28: 241-251.

Hoppenreijs, T. J., Nijdam, E. S. \& Freihofer, H. P. (1992) The chin as a donor site in early secondary osteoplasty: A retrospective clinical and radiological evaluation. Journal of Cranio-Maxillo-Facial Surgery 20: 119-124.

Jensen, J. \& Sindet-Pedersen, S. (1991) Autogenous mandibular bone grafts and osseointegrated implants for reconstruction of the severely atrophied maxilla: A preliminary report. Journal of Oral and Maxillofacial Surgery 49: 1277-1287.

Jensen, S. S. \& Terheyden, H. (2009) Bone augmentation procedures in localized defects in the alveolar ridge: Clinical results with different bone grafts and bone-substitute materials. International Journal of Oral and Maxillofacial Implants 24 Suppl: 218-236.

Lundgren, S., Moy, P., Johansson, C. \& Nilsson, H. (1996) Augmentation of the maxillary sinus floor with particulated mandible: A histologic and histomorphometric study. International Journal of Oral and Maxillofacial Implants 11: 760-766.

31 Makris, N., Stamatakis, H., Syriopoulos, K., Tsiklakis, K. \& van der Stelt, P. F. (2010) Evaluation of the visibility and the course of the mandibular incisive canal and the lingual foramen using cone-beam computed tomography. Clinical Oral Implants Research 21: 766-771.

34 Misch, C. M. (1997) Comparison of intraoral donor sites for onlay grafting prior to implant placement. International Journal of Oral and Maxillofacial Implants 12: 767-776. 
Nkenke, E. \& Neukam, F. W. (2014) Autogenous bone harvesting and grafting in advanced jaw resorption: Morbidity, resorption and implant survival. European Journal of Oral Implantology 7 Suppl 2: S203-217.

Nkenke, E., Schultze-Mosgau, S., Radespiel-Troger, M., Kloss, F. \& Neukam, F. W. (2001) Morbidity of harvesting of chin grafts: A prospective study. Clinical Oral Implants Research 12: 495-502.

Pereira, C. C., Gealh, W. C., Meorin-Nogueira, L., Garcia-Junior, I. R. \& Okamoto, R. (2014) Piezosurgery applied to implant dentistry: Clinical and biological aspects. Journal of Oral Implantology 40 Spec No: 401-408.

Raghoebar, G. M., Louwerse, C., Kalk, W. W. \& Vissink, A. (2001) Morbidity of chin bone harvesting. Clinical Oral Implants Research 12: 503-507.

Romanos, G. E., Gupta, B., Davids, R., Damouras, M. \& Crespi, R. (2012) Distribution of endosseous bony canals in the mandibular symphysis as detected with cone beam computed tomography. International Journal of Oral and Maxillofacial Implants 27: 273-277.

Silva, F. M., Cortez, A. L., Moreira, R. W. \& Mazzonetto, R. (2006) Complications of intraoral donor site for bone grafting prior to implant placement. Implant Dentistry 15: 420-426.

Soehardi, A., Meijer, G. J., Strooband, V. F., de Koning, M. \& Stoelinga, P. J. (2009) The potential of the horizontal ramus of the mandible as a donor site for block and particular grafts in pre-implant surgery. International Journal of Oral and Maxillofacial Surgery 38: 1173-1178.

Sohn, D. S., Ahn, M. R., Lee, W. H., Yeo, D. S. \& Lim, S. Y. (2007) Piezoelectric osteotomy for intraoral harvesting of bone blocks. International Journal of Periodontics and Restorative Dentistry 27: 127-131.

Verdugo, F., Simonian, K., Raffaelli, L. \& D'Addona, A. (2014) Computer-aided design evaluation of harvestable mandibular bone volume: A clinical and tomographic human study. Clinical Implant Dentistry and Related Research 16: 348-355.

von Arx, T., Hafliger, J. \& Chappuis, V. (2005) Neurosensory disturbances following bone harvesting in the symphysis: A prospective clinical study. Clinical Oral Implants Research 16: 432-439. harvesting--a retrospective long-term follow-up study. Clinical Implant Dentistry and Related Research 11: $149-157$. tomography. Dent Traumatol 25: 475-479. 


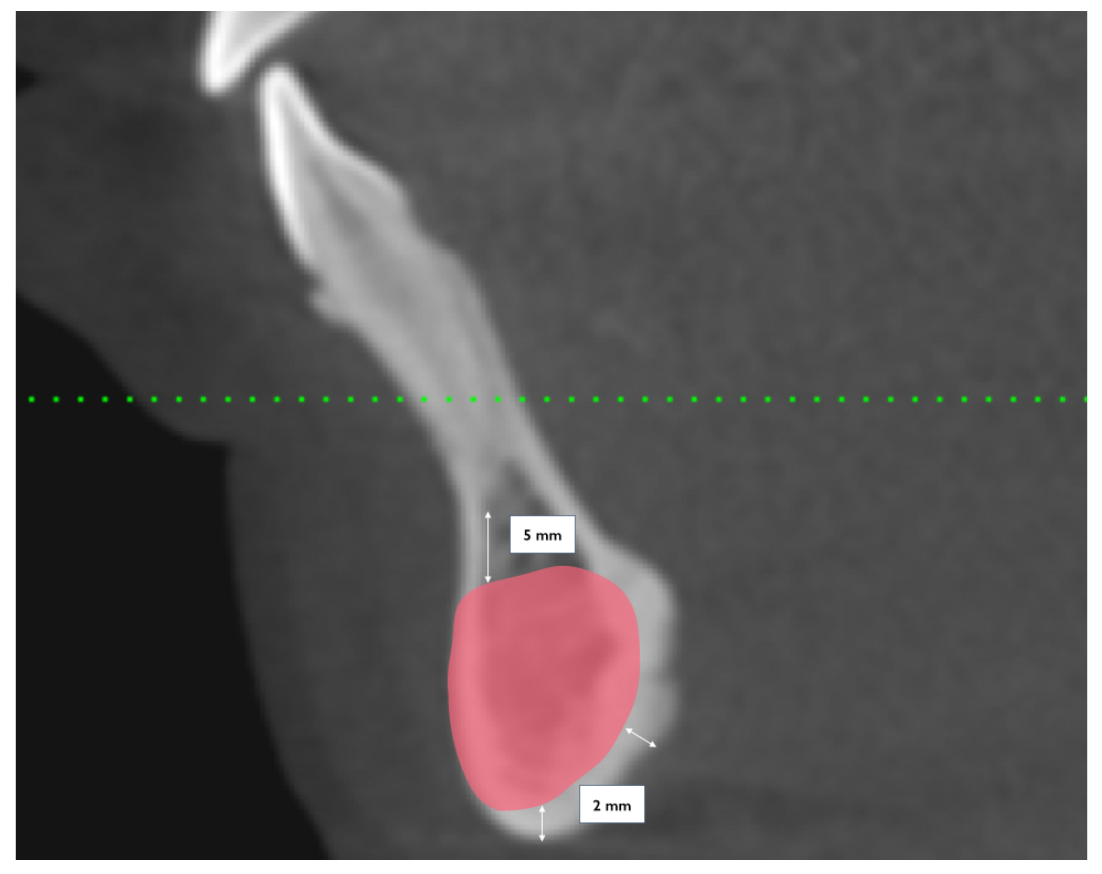

Figure 1

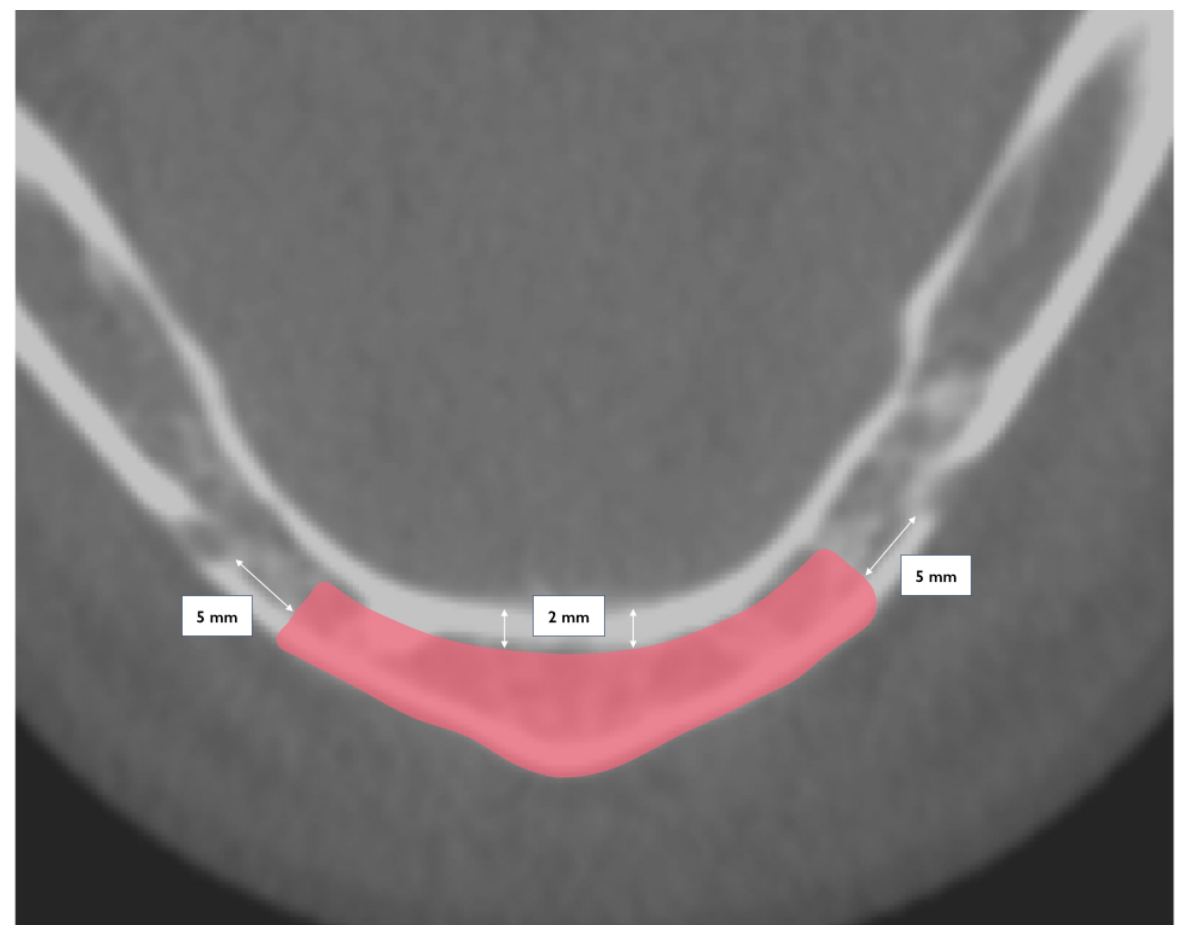

Figure 2 


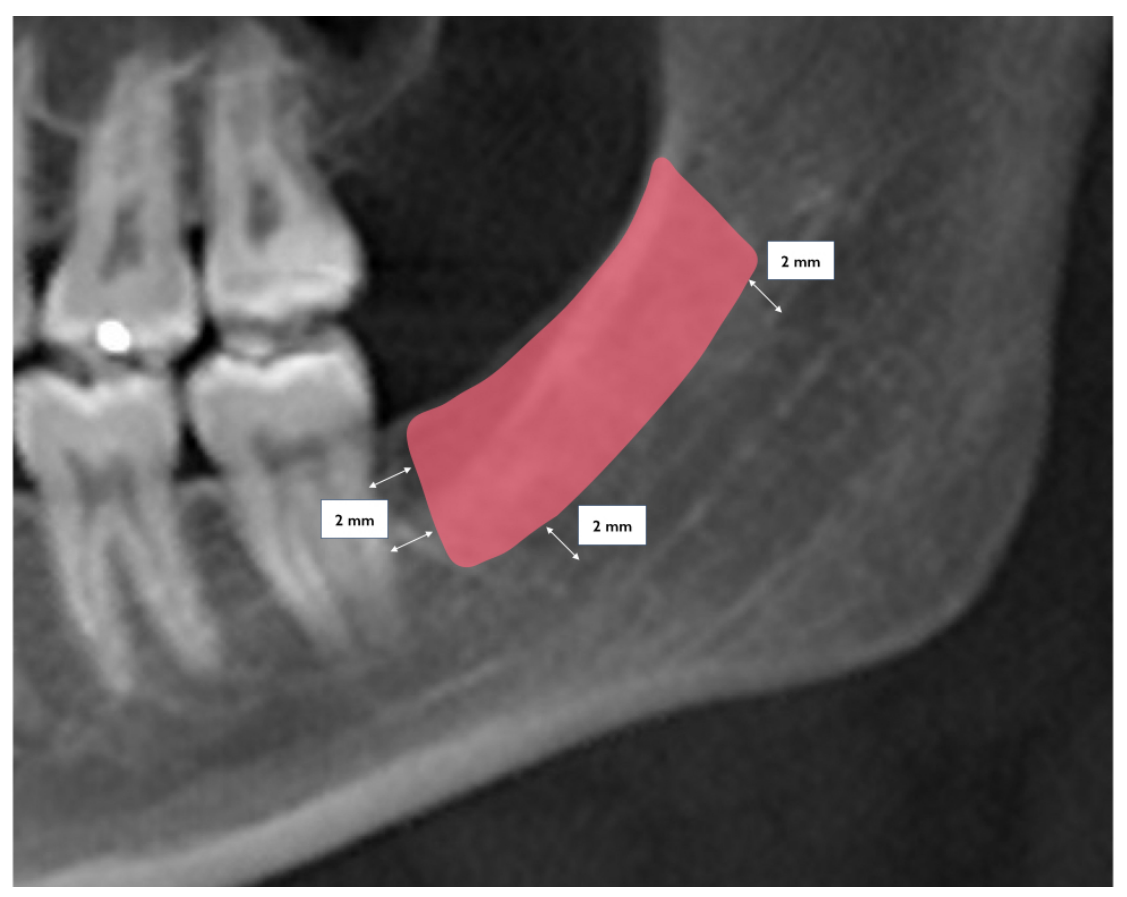

Figure 3

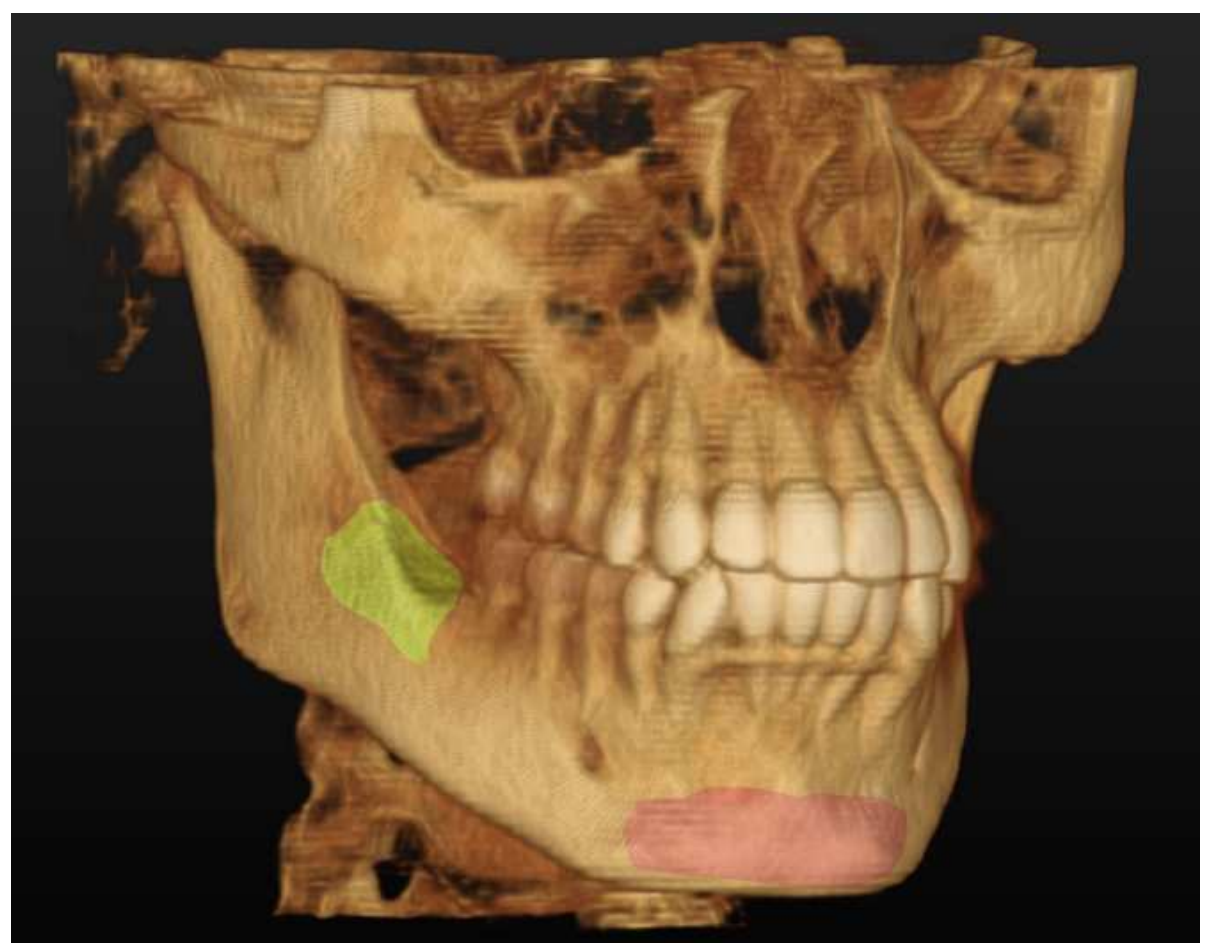

Figure 4 
Table 1. Results of volume, length, width and height of donor sites in the chin and the retromolar region for different study groups

\begin{tabular}{|c|c|c|c|c|c|c|c|c|c|c|c|c|c|c|c|c|c|c|c|c|c|}
\hline \multirow[b]{2}{*}{ Parameter } & \multirow[b]{2}{*}{ Group } & \multirow[b]{2}{*}{$\mathrm{n}$} & \multicolumn{6}{|c|}{ Chin } & \multicolumn{6}{|c|}{ Retromolar } & \multicolumn{6}{|c|}{ Ratio } & \multirow[b]{2}{*}{$p$-value* } \\
\hline & & & $\overline{M e a n} \pm$ SD & Min & Max & Q1 & Median & $\mathrm{Q} 2$ & Mean \pm SD & Min & Max & Q1 & Median & Q2 & $\overline{M e a n} \pm$ SD & Min & Max & Q1 & Median & Q2 & \\
\hline \multirow[t]{3}{*}{ Volume $\left(\mathrm{cm}^{3}\right)$} & M1 & 20 & $3.42 \pm 1.52$ & 0.59 & 6.57 & 2.46 & 3.39 & 4.31 & $2.58 \pm 1.43$ & 0.62 & $\overline{6.14}$ & 1.65 & 2.44 & 3.04 & $0.82 \pm 0.39$ & 0.25 & 1.86 & 0.54 & 0.79 & 1.04 & $0.036+$ \\
\hline & M2 & 20 & $3.42 \pm 0.93$ & 1.93 & 6.14 & 2.82 & 3.37 & 3.91 & $1.83 \pm 0.54$ & 1.01 & 2.96 & 1.44 & 1.82 & 2.19 & $0.57 \pm 0.20$ & 0.23 & 1.03 & 0.42 & 0.58 & 0.69 & $<0.001+$ \\
\hline & M3 & 20 & $3.63 \pm 1.40$ & 1.48 & 6.57 & 2.57 & 3.31 & 4.68 & $1.05 \pm 0.35$ & 0.34 & 1.83 & 0.79 & 1.04 & 1.33 & $0.32 \pm 0.12$ & 0.07 & 0.54 & 0.23 & 0.30 & 0.40 & $<0.001 \dagger$ \\
\hline \multirow[t]{3}{*}{ Length (mm) } & M1 & 20 & $35.12 \pm 4.64$ & 23.36 & 45.05 & 33.85 & 35.35 & 37.11 & $34.21 \pm 6.47$ & 23.29 & 45.55 & 28.44 & 34.95 & 36.47 & $0.99 \pm 0.25$ & 0.69 & 1.76 & 0.80 & 0.93 & 1.18 & $0.180^{\circ}$ \\
\hline & M2 & 20 & $37.32 \pm 3.51$ & 31.03 & 41.90 & 35.12 & 37.76 & 40.54 & $24.75 \pm 4.33$ & 17.35 & 31.58 & 20.93 & 26.13 & 27.77 & $0.67 \pm 0.15$ & 0.46 & 0.95 & 0.55 & 0.67 & 0.78 & $<0.001 \dagger$ \\
\hline & M3 & 20 & $39.95 \pm 3.37$ & 33.66 & 47.20 & 37.86 & 40.51 & 42.05 & $15.53 \pm 3.40$ & 7.27 & 22.52 & 13.70 & 15.41 & 18.29 & $0.39 \pm 0.10$ & 0.19 & 0.56 & 0.33 & 0.41 & 0.46 & $<0.001 \dagger$ \\
\hline \multirow[t]{3}{*}{ Width (mm) } & M1 & 20 & $10.20 \pm 2.10$ & 6.10 & 14.62 & 8.61 & 9.79 & 12.08 & $8.95 \pm 1.48$ & 6.35 & 11.61 & 8.15 & 9.09 & 9.74 & $0.90 \pm 0.17$ & 0.60 & 1.24 & 0.77 & 0.90 & 0.99 & $0.017{ }^{\dagger}$ \\
\hline & M2 & 20 & $10.60 \pm 1.88$ & 7.54 & 14.72 & 9.01 & 10.86 & 11.71 & $8.50 \pm 1.32$ & 5.28 & 10.09 & 7.85 & 8.50 & 9.60 & $0.82 \pm 0.19$ & 0.51 & 1.21 & 0.70 & 0.80 & 0.91 & $0.001+$ \\
\hline & M3 & 20 & $10.00 \pm 2.30$ & 5.77 & 14.18 & 8.34 & 10.15 & 11.59 & $8.03 \pm 1.60$ & 4.62 & 12.03 & 7.23 & 7.89 & 8.96 & $0.84 \pm 0.22$ & 0.45 & 1.34 & 0.69 & 0.82 & 0.93 & $0.007+$ \\
\hline \multirow[t]{3}{*}{ Height (mm) } & M1 & 20 & $13.04 \pm 4.02$ & 2.80 & 19.26 & 10.56 & 13.01 & 15.91 & $10.19 \pm 1.97$ & 6.96 & 13.95 & 8.56 & 9.57 & 12.00 & $0.91 \pm 0.57$ & 0.48 & 3.10 & 0.64 & 0.70 & 1.09 & $0.074^{\circ}$ \\
\hline & M2 & 20 & $12.64 \pm 2.96$ & 6.88 & 19.23 & 10.39 & 13.24 & 14.45 & $10.45 \pm 1.82$ & 5.86 & 13.30 & 9.58 & 10.40 & 12.09 & $0.87 \pm 0.26$ & 0.42 & 1.52 & 0.70 & 0.77 & 1.06 & $0.115^{\circ}$ \\
\hline & M3 & 20 & $12.45 \pm 2.76$ & 7.66 & 18.53 & 11.42 & 12.04 & 14.21 & $10.41 \pm 2.09$ & 6.38 & 13.49 & 8.58 & 10.89 & 12.26 & $0.87 \pm 0.21$ & 0.44 & 1.30 & 0.72 & 0.90 & 1.00 & $0.011^{\dagger}$ \\
\hline
\end{tabular}

